\title{
Philosophiques
}

\section{Sur la théorie des démonstrations}

\section{Y. Gauthier}

Volume 8, numéro 2, octobre 1981

URI : https://id.erudit.org/iderudit/203170ar

DOI : https://doi.org/10.7202/203170ar

Aller au sommaire du numéro

Éditeur(s)

Société de philosophie du Québec

ISSN

0316-2923 (imprimé)

1492-1391 (numérique)

Découvrir la revue

Citer cet article

Gauthier, Y. (1981). Sur la théorie des démonstrations. Philosophiques, 8(2), 273-285. https://doi.org/10.7202/203170ar

\section{Résumé de l'article}

Le texte est consacré aux aspects essentiels de la théorie des démonstrations en logique mathématique et à ses ramifications contemporaines. La distinction établie par Kreisel entre théorie générale des démonstrations et théorie reductive des démonstrations est reprise et l'accent est mis sur la théorie reductive ou les sous-systèmes de l'analyse classique, en particulier l'induction transfinie. Le texte comporte une critique de la justification de l'induction transfinie de Takeuti et se termine par une liste des développements contemporains les plus significatifs en théorie de la preuve. d'utilisation que vous pouvez consulter en ligne.

https://apropos.erudit.org/fr/usagers/politique-dutilisation/ 


\section{SUR LA THÉORIE DES DÉMONSTRATIONS*}

RÉSUMÉ. Le texte est consacré aux aspects essentiels de la théorie des démonstrations en logique mathématique et à ses ramifications contemporaines. La distinction établie par Kreisel entre théorie générale des démonstrations et théorie réductive des démonstrations est reprise et l'arcent est mis sur la théorie réductive ou les sous-systèmes de l'analyse classique, en particulier l'induction transfinie. Le texte comporte une critique de la justification de l'induction transfinie de Takeuti et se termine par une liste des développements contemporains les plus significatifs en théorie de la preuve.

ABSTRACT. This article deals with the essential aspects and the contemporary developments of proof theory in mathematical logic. The distinction between general proof theory and reductive proof theory borrowed from Kreisel is used as the main axis of the paper, but the emphasis is on the reductive theory of classical analysis and transfinite induction. A foundational critique of Takeuti's justification of transfinite induction is offered and details on the most significant contemporary developments of proof theory close the article.

Nous nous proposons, dans ce court texte, de présenter les aspects essentiels de la théorie des démonstrations et ses ramifications contemporaines. Nous faisons aussi une critique de la notion d'induction transfinie, centrale dans la théorie mathématique des démonstrations. Enfin, nous défendons une position fondationnelle, le constructivisme, que l'on trouvera plus étayée ailleurs.

La théorie des démonstrations, ou de la preuve, a son origine chez Hilbert qui, le premier, en a fait une discipline indépen-

\footnotetext{
* Ce texte a fait l'objet d'une conférence aux départements de mathématiques et de philosophie de l'Université du Québec à Trois-Rivières en février 1980, conférence qui a aussi été donnée dans le cadre du séminaire «Logique et épistémologie» du département de philosophie de l'Université du Québec à Montréal. Nous remercions Daniel Vanderveken et Serge Robert pour leurs invitations respectives.
} 
dante comme Beweistheorie ou encore Metamathematik, c'està-dire étude des systèmes formels des théories mathématiques. Le problème qui motivait Hilbert était celui de la non-contradiction de l'analyse classique, problème qui n'est pas encore résolu aujourd'hui. Mais depuis Hilbert, la théorie des démonstrations s'est diversifiée considérablement et constitue, avec la théorie des modèles, l'une des deux artères principales de la logique mathématique. Si la théorie des modèles s'occupe de la sémantique, c'est-à-dire de l'interprétation des théories logiques et mathématiques, la théorie des démonstrations s'intéresse à leur seule syntaxe ou structure formelle (la pragmatique ressortit, elle, à la sémantique).

Pour les besoins de cet article, nous distinguerons la théorie générale des démonstrations, qui relève de la logique formelle, de la théorie réductive des démonstrations, qui fait partie de la logique mathématique. ${ }^{1}$

1. Depuis Euclide, la démonstration fait partie intégrante des mathématiques. Mais l'intérêt qu'on accorde à l'élaboration d'une preuve n'a pas toujours été égal: ainsi le célèbre mathématicien anglais G.H. Hardy ne dit-il pas que "les preuves ne sont rien, seuls les bons concepts comptent ${ }^{2} »$. Il est sans doute vrai que les mathématiciens eux-mêmes se répartissent en deux classes, les "concepteurs" ou créateurs de concepts (et de théories parfois) et les «prouveurs» (ou «démonstrateurs»), qui démontrent des théorèmes qu'ils n'ont pas conçus eux-mêmes. Le couple Cantor-Dedekind vient de suite à l'esprit; Cantor avouait qu'il n'était certain d'une preuve que lorsqu'il l'avait soumise à Dedekind dont il disait qu'il avait un esprit en escalier, Treppenverstand $^{3}$. Dedekind était par ailleurs, lui aussi, un mathématicien créateur. Des mathématiciens comme Thom (la théorie du cobordisme en topologie différentielle qu'il a créée de toutes pièces n'est pas encore entièrement démontrée) ou Grothendieck (pour

1. Cette distinction est due à Georg Kreisel et a été reprise par D. Prawitz dans «Ideas and Results in Proof Theory", Proceedings of the Second Scandinavian Logic Colloquium, NorthHolland, Amsterdam, 1971, pp. 235-307. On consultera aussi G. Kreisel «A Survey of Proof Theory II" Ibid., pp. 109-170 dont la première partie est parue dans The Journal of Symbolic Logic, vol. 33 (1968), pp. 321-388.

2. Voir G.H. Harvy, A Matbematician's Apology. London, Cambridge University Press, 1940.

3. Voir la correspondance Cantor-Dedekind dans G. Cantor, Gesammelte Abhandlungen, hrsg, v. E. Zermelo, G. Olms, Hildesheim, 1966. 
la géométrie algébrique) sont des créateurs, alors qu'un Deligne par exemple serait un "prouveur». En logique, on pourrait penser à Kreisel et à Cohen. Mais ce n'est là que la psychologie des mathématiques qui n'a pas d'intérêt immédiat pour nous.

Bourbaki est l'un (ou plusieurs!) de ceux qui prennent la preuve au sérieux. «Qui dit mathématique, dit démonstration» déclare-t-il au tout début de son entreprise ${ }^{4}$. Qui dit démonstration, dit logique, pourrions-nous ajouter. Montrons-le tout de suite.

Bourbaki énumère quatre types de preuve dans son traité: a) preuve par hypothèse auxiliaire; b) preuve par réduction à l'absurde; c) preuve par disjonction des cas et d) preuve par constante auxiliaire. Il n'est pas difficile de donner la version formelle de ces types de preuve: la preuve par hypothèse auxiliaire où l'énoncé $A$ est supposé vrai (ou est un axiome) correspond à la règle d'introduction du fer à cheval I $\supset$

$$
\begin{gathered}
{[A]} \\
B D
\end{gathered}
$$

la preuve indirecte ou reductio ad absurdum où l'on suppose que $A$ est faux correspond à la règle d'élimination de la négation $\mathrm{E}$

$$
\begin{aligned}
& {[\sim \mathrm{A}] \quad \text { (pour la logique classique, s'entend) }} \\
& -\Lambda-
\end{aligned}
$$

( $\Lambda$ signifie contradiction), la preuve par disjonction des cas où de $A \vee B$ il faut passer par $A \supset C$ et $B \supset C$ pour obtenir $C$ correspond à la règle d'élimination du «disjoncteur» $\mathrm{E} V$

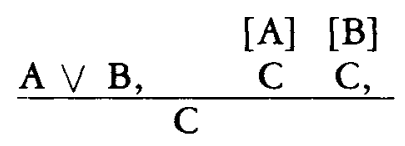

enfin la preuve par la constante auxiliaire où l'on suppose que l'on a un $\mathrm{x}$ tel que $\mathrm{A}$ (prédicat) correspond à la règle d'introduction du quantificateur universel IV

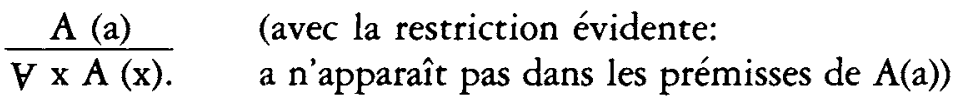

4. Cf. N. Bourbaki, Éléments des mathématiques. Théorie des ensembles. Hermann, Paris, 1970. 
Ces règles dites de déduction naturelle ont été formulées par Gentzen et font partie de l'arsenal de la logique formelle contemporaine ${ }^{5}$. Outre la règle d'inférence modus ponens connue depuis Aristote et qui correspond à la règle d'élimination du fer à cheval E $\supset$

$$
\frac{A, A \supset B}{B}
$$

on a la règle de coupure

$$
\frac{\mathrm{A} \supset \mathrm{D}, \mathrm{D} \supset \mathrm{B}}{\mathrm{A} \supset \mathrm{B}}
$$

qui joue un rôle important dans les théorèmes de forme normale qui garantissent qu'on peut obtenir une dérivation sans la règle de coupure dans un système formel donné - en plus des théorèmes de forme normale, on a des théorèmes de normalisation qui garantissent que toute dérivation est normalisable. Gentzen, Schütte, Prawitz, Martin-Löf, Girard ont obtenu ces résultats pour divers systèmes formels, e.g. logique des prédicats de premier ordre, du second ordre, théorie des types, arithmétique, etc. L'important principe d'inversion (qu'on attribue à Lorenzen) stipule que la conclusion obtenue par une règle d'élimination ne dit rien de plus que ce qui aurait été acquis si la prémisse majeure de l'élimination avait été inférée par une règle d'introduction ${ }^{6}$.

2. La théorie de la déduction naturelle appartient à la théorie générale des démonstrations; le coeur de la théorie des démonstrations est cependant la théorie «réductive» - cette épithète signifie que l'on cherche à formuler des sous-systèmes, des systèmes formels «réduits» de plus en plus forts pour tenter $\mathrm{d}$ '«approximer» le problème de la consistance de l'analyse classique ou de la théorie des ensembles, demeuré inaccessible jusqu'à maintenant. Ces sous-systèmes de l'analyse sont, par exemple, les hiérarchies arithmétique, hyperarithmétique et analytique $\sum_{n}^{0}$, $\prod_{n}^{0}$, (où l'on quantifie sur des variables numériques et des variables fonctionnelles respectivement) en théorie de la récursion ou

5. Pour une présentation plus complète de ces règles, nous nous permettons de renvoyer à notre Méthodes et concepts de la logique formelle. Montréal, Presses de l'Université de Montréal, 1978.

6. Voir D. Prawitz, Natural Deduction, Almquist \& Wiksell, Stockholm, 1965. 
des versions affaiblies de l'arithmétique du second ordre $<\mathrm{N}, \mathrm{O}$, $\mathrm{S},=,+, \cdot>$ avec le postulat d'induction

$$
\mathrm{V} \mathrm{X}[\mathrm{XO} \wedge \mathrm{Vy}(\mathrm{Xy} \rightarrow \mathrm{XSy}) \rightarrow \mathrm{VyXy}] .^{?}
$$

Mais l'instrument principal ici est l'induction transfinie jusqu'à $\epsilon_{0}$, c'est-à-dire la limite des $\omega$. Voyons maintenant ce qu'il en est de cette induction transfinie. On sait que Cantor, le créateur de la théorie des ensembles transfinis, a établi la hiérarchie des ordinaux suivante

$$
\begin{aligned}
\omega & =\lim <0,1,2, \ldots, \mathrm{n}\rangle \\
\omega \cdot 2 & =\lim <\omega+\mathrm{n}\rangle \\
\omega^{2} & =\lim <\omega \cdot \mathrm{n}\rangle \\
\omega^{\omega} & =\lim \left\langle\omega^{\mathrm{n}}>\right. \\
\omega^{\omega^{\omega}} & \left.=\lim <\omega^{\omega^{\mathrm{n}}}\right\rangle \\
\epsilon_{0} & =\lim \left\langle\omega^{\omega^{\cdot}}, \mathrm{n}\right\rangle
\end{aligned}
$$

(un ordinal limite est un ordinal qui n'a pas de prédécesseur immédiat). On remarque que chacun des ensembles ordonnés de la hiérarchie a pour dernier terme n puisque la hiérarchie est fondée sur la forme normale que Cantor a donnée pour tout ordinal

$$
\xi=\omega^{\beta_{n_{n_{1}}}}+\omega^{\beta_{\mathrm{n}_{\mathrm{n}_{2}}}}+\ldots+\omega^{\beta_{\mathrm{m}_{\mathrm{n}_{m}}}}
$$

où $\beta_{1}>\beta_{2}>\ldots>\beta_{\mathrm{m}}$ et $\mathrm{m}, \mathrm{n}_{1}, \mathrm{n}_{2}, \ldots, \mathrm{n}_{\mathrm{n}}$ sont finis. C'est la deuxième classe de nombres de Cantor aussi appelés «ordinaux constructifs» par Kleene et Church; cette classe est, en effet, dénombrable et récursivement énumérable au sens de la théorie des ensembles à cause du terme $\mathrm{n}$, mais n'est pas effectivement énumérable au sens constructiviste (et au sens strict) puisqu'on peut trouver un ordinal tel que $\alpha_{\mathrm{n}}=\alpha$ pour tous les n: on n'a qu'à prendre $\alpha=\lim \alpha_{\mathrm{n}}$, donc $\alpha_{\mathrm{n}}<\alpha$. On voit que les concepts sont assez fins ici; la thèse de Church affirme qu'un ensemble récursivement énumérable est aussi effectivement énumérable (on vient de voir que ce n'est pas le cas) ou encore qu'une fonction récursive est équivalente à une prescription qui génère un à un les éléments d'un ensemble. Il est bien connu que l'ensemble des fonctions récursives primitives est équivalent à toute une série de systèmes

7. Pour plus de détails sur ces questions, on pourra consulter notre Fondements des matbématiques. Montréal, Presses de l'Université de Montréal, 1976. 
formels, machine de Turing, $\lambda$-définissabilité, calcul équationnel, systèmes canoniques de Post, algorithmes normaux de Markov. Mais par la méthode de diagonalisation de Cantor, je peux trouver dans $\mathrm{N}^{\mathrm{N}}$ une fonction distincte de toutes les fonctions calculables (donc récursives, par la thèse de Church) et qui n'est pas, par conséquent, calculable: je n'ai qu’à me donner une énumération (effective, par définition) des fonctions calculables et je définis

$$
g(n)=\left\{\begin{array}{l}
1, \text { ssi } f_{n}(n)=0 \\
0, \text { autrement; }
\end{array}\right.
$$

$g$ n'est pas calculable, parce que pour un $k$ arbitraire $g(k)=f_{k}(k)$, mais $\mathrm{f}_{\mathrm{k}}(\mathrm{k})=0$, ssi $\mathrm{g}(\mathrm{k})=1$, donc $\mathrm{g}(\mathrm{k}) \neq \mathrm{f}_{\mathrm{k}}(\mathrm{k}){ }^{8}$ Inutile de dire que le procédé de diagonalisation n'est pas constructif lui non plus...

C'est dans sa preuve de non-contradiction de l'arithmétique (1936) que Gentzen utilise l'induction transfinie. Hilbert avait voulu se limiter à une preuve de non-contradiction "finie», c'est-à-dire à une configuration finie d'objets concrets, de signes formels; c'est la combinatoire finie à laquelle Hilbert pensait pouvoir réduire la théorie de la preuve. La seconde preuve d'incomplétude (1931) de Gödel modifiera sensiblement cet idéal. L'outil majeur de Hilbert était le symbole $\epsilon$, une fonction de choix (approximative) employée un nombre fini de fois et qui permettait d'éliminer les quantificateurs:

$$
\begin{aligned}
A(t) & \rightarrow A\left(\epsilon_{x} A(x)\right) \\
\exists x A(x) & =A\left(\epsilon_{x} A(x)\right) \\
\forall x A(x) & =A\left(\epsilon_{x} \sim A(x)\right)^{9}
\end{aligned}
$$

Puis Skolem introduit la notion d'expansion, c'est-à-dire conjonction de formules avec instantiation existentielle mais sans quantificateurs - technique qui allait mener à ce qu'on appelle élimination des quantificateurs. Le théorème de Herbrand utilise cette notion d'expansion de façon syntaxique: une formule est dérivable, si sa négation a une expansion inconsistante en termes de valeurs de vérité. D'autre part Skolem, Gödel, Tarski créaient la

8. Nous reprenons un passage de notre Méthodes et concepts de la logique formelle, p. 132.

9. On consultera là-dessus D. Hilbert et P. Bernays, Grundlagen der Mathematik, vol. 2, Berlin, Springer, 1939, qui contient les détails sur les méthodes utilisant le symbole $\epsilon$. 
théorie des modèles avec la notion centrale de satisfaction (d'une formule ou d'un ensemble de formules).

Après le résultat de Gödel, qui démontrait que la preuve de consistance d'une théorie devait utiliser des moyens (formels) plus forts que ceux dont disposait le système formel de la théorie en question, il était évident qu'il fallait aller au-delà de la combinatoire finie de Hilbert. L'intuitionnisme de Brouwer comportait déjà des objets abstraits, fonctions, fonctionnelles, constructions, preuves qui transcendaient le point de vue strictement finitaire de Hilbert, mais cette voie ne sera exploitée que beaucoup plus tard par Gödel dans son interprétation «Dialectica» ${ }^{10}$. Pour Gentzen, l'induction transfinie signifiait le parcours ou la traversée «potentielle» d'une totalité infinie. Gentzen a toujours insisté sur cette interprétation «potentialiste» ${ }^{11}$, mais le Gedankenexperiment du parcours potentiel d'un ensemble infini est inconsistant; nous allons le montrer en critiquant la justification de l'induction transfinie qu'a voulue donner Takeuti dans son livre Proof Theory ${ }^{12}$. Takeuti part du principe suivant: «quand tous les nombres plus petits que $\beta$ sont reconnus comme accessibles, alors $\beta$ est lui-même accessible», mais au lieu de suites strictement croissantes d'ordinaux $\beta_{0}<\beta_{1}<\ldots<\beta \epsilon_{0}$, il introduit des suites strictement décroissantes $\mu>\ldots>\mu_{1}>\mu_{0}$ pour $\mu=\lim \left(\omega_{n}\right)$, et tente de montrer par une méthode concrète que toute suite est finie. L'idée se résume à celle des arbres bien fondés (ou bien enracinés)

10. K. Gödel, «Ueber eine bis jetzt noch nicht benützte Erweiterung des finiten Standpunktes», Dialectica 12 (1958), pp. 280-287.

11. G. Gentzen Collected papers, ed. by E. Szabo, North-Holland, Amsterdam, 1969.

12. G. Takeuti, Proof Theory, North-Holland, Amsterdam, 1975, chap. 2. On pourra consulter aussi sur la théorie des démonstrations, l'ouvrage classique de K. Schütte Beweistheorie. Berlin, Springer, 1960, mais Schütte ne tente pas de justifier l'induction transfinie. La traduction en anglais comporte de nombreux résultats de la théorie des démonstrations (e.g. systèmes formels de l'arithmétique, systèmes semi-formels, etc.), mais rien de neuf fondationnellement parlant (cf. K. Schütte, Proof theory. New York, Springer, 1977). 


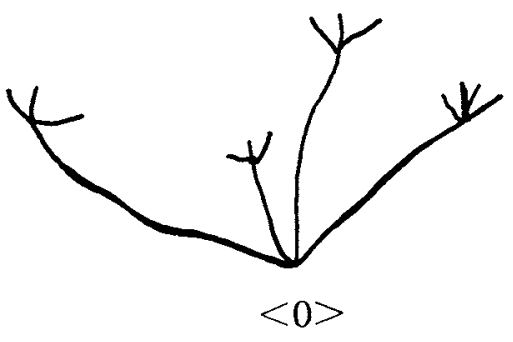

ou

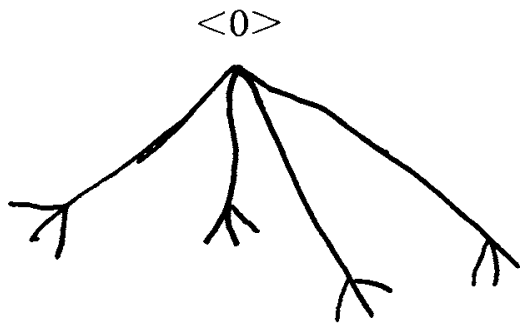

qui illustrent le concept de suite finie: tout sentier ou toute branche est finie puisqu'on peut y remonter ou y redescendre en un temps fini jusqu'au premier ordinal 0 .

Takeuti conclut donc: si $\mu_{1}+\mu_{2}+\ldots+\mu_{n}$ sont des ordinaux, alors $\mu_{1}+\mu_{2}+\ldots+\mu_{n}$ et $\omega^{\mu}$ en sont avec $0, \omega^{0}=1$ et $\mu=\lim \omega^{\mu_{1}}$ ) et formule la proposition suivante:

Proposition I: «un ordinal $\mu$ est accessible s'il a été démontré que toute suite strictement décroissante commençant par $\mu$ est finie».

Puisqu'il s'agit de suites strictement décroissantes, nous préférons parler d'ordinaux «récessibles» et par analogie avec le théorème de Heine-Borel sur la continuité uniforme sur un intervalle borné fermé, nous introduisons la notion de récessibilité uniforme et nous énonçons la proposition suivante:

Proposition II: «un ordinal $\mu$ est uniformément récessible s'il a été démontré que dans toute suite strictement décroissante tout $\mu_{\mathrm{n}}$ a un récesseur immédiat»

De cette proposition nous tirons immédiatement le théorème suivant: Théorème: « $\epsilon_{0}$ n'est pas uniformément récessible». 
Preuve: la suite des ordinaux de la deuxième classe de Cantor comporte des termes ou points singuliers ou de discontinuité à chaque ordinal limite de la suite - c'est-à-dire, aucun des $\omega$, $\omega \cdot 2, \omega^{2}, \omega^{\omega}, \omega^{\omega^{\omega}}, \epsilon_{0}$ n'a de récesseur immédiat, donc $\epsilon_{0}$ n'est pas uniformément récessible $\square$.

Il est clair, par conséquent, qu'un ordinal qui n'est pas uniformément récessible est défini imprédicativement, c'està-dire par en haut, par rapport à une totalité à laquelle il appartient téléologiquement et non par en bas, par rapport à la génération sucessive de ses prédécesseurs. ${ }^{13} \mathrm{La}$ mal vient de l'«ensemblage", de la mise en ensembles de suites qui sont générées par itération et non par englobement ou totalisation. La suite des nombres naturels, par exemple, est effinie, c'est-à-dire n'a pas de dernier terme; il est illusoire de "globaliser" cette suite et d'en faire un «ensemble» infini et la confusion s'installe au moment où l'on attribue un ordinal à cette totalité des ordinaux (finis). Le passage de l' «ordination» à l' «ensemblage» est un procès «transarithmétique» comme nous l'avons appelé ailleurs et ce passage est illégitime d'un point de vue constructiviste (effinitaire).

Même si Gentzen, Takeuti et d'autres ont tenté de justifier l'induction transfinie en recourant à une interprétation "potentialiste» ou presque finitaire - on utilise toujours l'induction transfinie ou la bar-induction transfinie dans la métathéorie des systèmes formels de l'analyse intuitionniste par exemple - la notion d'induction transfinie n'a pas de contenu constructif en vertu du caractère imprédicatif de la notion d'ensemble infini achevé ou clos; cette clôture interdit en principe de franchir le premier $\omega$...

3. La dinstinction entre preuves constructives et preuves non constructives est la plus nette en théorie des nombres (la théorie mathématique des nombres et non la théorie logique ou arithmétique du premier et du second ordre). Depuis longtemps on distingue une preuve constructive ou élémentaire effectuable

13. La même critique s'applique à la notion d'échelle récursive primitive telle qu'exploitée par Girard dans "A survey of $\pi_{2}^{1}-\operatorname{logic}$ " in Abstracts of the $6^{\text {th }}$ International Congress of Logic Methodology and Philosophy of Science, Hannover, 1979 section I, pp. 2-6. La seule défense possible, c'est de dire comme Girard que la notion d'échelle transfinie ou d'ensemble infini doit être prise et comprise telle quelle, qu'elle n'est pas réductible (en particulier, pour la notion d'échelle, qu'elle peut être comprise sans la notion d'échelons!). 
en un nombre fini d'étapes d'une preuve analytique ou transcendante qui fait intervenir, outre le tiers exclu, des ensembles infinis comme les fonctions entières ou le prolongement analytique d'une fonction, etc. On sait que Bishop, qui a voulu, à l'instar de Brouwer, donner des fondements constructivistes à l'analyse (cf. Foundations of constructive Analysis), défend une interprétation numérique des mathématiques et rejette le tiers exclu sous la forme de ce que Bishop appelle le principe d'omniscience

d'où

$$
\forall \mathbf{P}(\mathrm{x}) \vee \mathbf{B} \mathrm{x} \sim \mathrm{P}(\mathrm{x})
$$

$$
\sim \mathrm{V} \mathrm{x} \mathrm{P}(\mathrm{x}) \supset \mathrm{Ax} \sim \mathrm{P}(\mathrm{x})
$$

Quant à l'intuitionnisme, qui a fait de la notion de preuve la notion centrale de son programme fondationnel, il rejette comme moyens de preuve le tiers exclu, les totalités infinies et toutes les constructions qui ne sont pas effectuables finiment. Le constructivisme radical que nous défendons - il y a plusieurs versions de constructivisme qui ne sont pas aussi radicales - que nous pourrions appeler "effinitaire», parce qu'il va au-delà du finitisme de Hilbert en admettant des suites «effinies», fonde les mathématiques et les méthodes de preuve légitimes sur des constructions finies, une quantification effinie $¥ \mathrm{x}$ pour les suites effinies et le concept de négation locale qui entraîne une réinterprétation constructiviste des constantes logiques et des concepts fondamentaux des mathématiques. Il ne s'agit pas ici de fonder la logique et les mathématiques sur l'intuition du temps comme dans l'intuitionnisme de Brouwer, sur des jeux de dialogue (Dialogspiele) comme chez Lorenzen ou sur une pratique discursive quelconque ou encore sur le langage ordinaire, mais plutôt sur une théorie des assertions et des négations (c'est-à-dire des domaines locaux d'assertion et de négation).

Une telle théorie entraîne des changements profonds de la logique et des mathématiques classiques. Mais nous ne pouvons en parler plus longuement $\mathrm{ici}^{14}$.

14. Nous avons commencé à formuler quelques théorèmes fondamentaux de cette théorie dans "Une théorie de la négation locale» qui constituera le premier chapitre d'un livre à paraître bientôt sous le titre Tbéorétiques. Pour une philosophie constructiviste des sciences. Ces résultats ont été annoncés dans "Vers une théorie de la négation locale» dans Abstracts of the $6^{\text {th }}$ International Congress of Logic, Metbodology and Pbilosophy of Science, Hannover, 1979, Section I, pp. $25-29$. 
4. Nous terminerons en indiquant brièvement les développements contemporains le plus significatifs de la théorie des démonstrations:

a) la théorie géométrique des preuves appelée par Kreisel «theory of proofs» pour la distinguer de la «proof theory». Cette théorie s'intéresse à la longueur des preuves (ou des dérivations), leur structure géométrique (e.g. leur genre) et tente de fixer des bornes à la complexité (travaux de Stadtman)

b) la théorie algébrique des preuves où l'on utilise des moyens algébriques et catégoriques (théorie des catégories) pour analyser la structure externe des preuves, e.g. longueur.

c) la théorie de la complexité s'occupe aussi des problèmes de longueur des preuves, mais dans un esprit plus arithmétique; on rencontre ici les problèmes décidables mais exponentiellement complexes, c'est-à-dire des problèmes dont on sait qu'il sont solubles en principes mais non en pratique à cause de leur complexité qui croît exponentiellement,

d) un domaine apparenté à celui-là, la théorie de la récursion généralisée et des algorithmes d'ordinateurs, est une extension de la théorie classique de la récursion associée aux travaux sur les algorithmes abstraits.

e) dans ce sens, un des développements les plus intéressants est la jonction de la théorie des démonstrations et de la théorie de l'information. Chaitin a obtenu un algorithme général $\Omega$ qui condense en quelque sorte tout le problème de l'indécidable; on a les données suivantes:

- M, une machine de Turing universelle

- $\mathrm{P}(\mathrm{s})$, la probabilité qu'elle s'arrête après un certain nombre (aléatoire) de coups de dés 0,1 ,

- $\mathrm{H}(\mathrm{s})$ est l'entropie; $\mathrm{H}(\mathrm{s})=-\log _{2} \mathrm{P}(\mathrm{s})$

- I(s) est la complexité du programme minimal ou le nombre minimal de bits (binary digits) requis pour spécifier l'algorithme qui commande à $\mathrm{M}$ de calculer $s$ ou l'information algorithmique

- $\Omega$ est le nombre réel qui représente la possibilité que $M$ s'arrête ${ }^{15}$.

15. Voir G.J. Chaitin "Algorithmic Information Theory" in IBM Journal of Research and Development, vol. 21, no. 4, pp. 350-359 (July 1977). 
C'est là une généralisation du théorème en théorie de la récursion qui démontre l'impossibilité d'une solution pour le problème de l'arrêt, c'est-à-dire il n'y a pas moyen de déterminer si un programme quelconque va stopper ou continuer indéfiniment; dans le cas de $\Omega$, c'est un aléatoire algorithmique «incompressible»: le nombre $\Omega$ est définissable, mais indémontrable et il l'est de plus en plus, peut-on dire, ou entropiquement, puisque le nombre de bits qu'il faut pour le définir est plus grand que le nombre de bits du système formel qu'il faut pour le démontrer. Cette généralisation du résultat de Gödel est optimale, dans le sens où on ne peut l'améliorer.

f) finalement on peut indiquer qu'on n'a pas épuisé l'analyse philosophique de la notion de preuve. Kreisel, après Hilbert, Bernays et d'autres, a insisté sur la nécessité d'une telle analyse. Les différentes sortes de preuves et les diverses méthodes de preuve ne sont pas réfractaires à l'examen, mais l'invasion de la sémantique ensembliste a brouillé bien des pistes (par exemple, la métalogique de la logique intuitionniste n'est pas souvent intuitionniste, alors que la métamathématique des mathématiques intuitionnistes l'est davantage). M. Dummett a proposé récemment une théorie de la signification fondée sur la notion intuitionniste de preuve qui n'a rien à voir avec les valeurs de vérité classiques ou encore la définition tarskienne de la vérité.

Nous avons suggéré ailleurs de distinguer trois moments

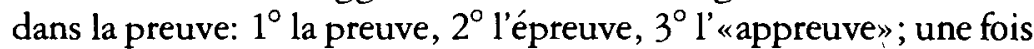
qu'une preuve est produite, il faut la vérifier ou la faire vérifier, l'évaluer (on peut alors l'améliorer en l'abrégeant, par exemple, ou en employant une autre voie démonstrative sans changer les moyens essentiels de la preuve). Un certain nombre de travaux ont été faits, par exemple, sur l'équivalence de deux preuves ou de deux dérivations si l'on tient compte de la distinction intuitionniste entre preuve et dérivation (où la preuve est un objet mental et la dérivation, sa représentation graphique). Le moment décisif demeure celui de l'appreuve, c'est-à-dire de l'approbation de la preuve et des moyens de sa démonstration. C'est ici qu'un constructiviste est le plus exigeant, mais il n'est pas seul à demander des comptes. Ainsi on a obtenu récemment la solution du problème classique des quatre couleurs en topologie - quatre couleurs suffisent pour colorier une carte planaire de telle sorte qu'il 
n'y ait pas deux pays limitrophes de la même couleur (Appel et Haken, 1976). La preuve fait appel à l'ordinateur à cause des longs calculs qui étaient nécessaires (1,200 heures).

Cependant, plusieurs mathématiciens ne sont pas convaincus du bien-fondé de la preuve et voudraient avoir une preuve plus courte, "faite à la main». Si l'appreuve ou l'approbation n'est pas acquise, la preuve perd son caractère de certitude - Hilbert parlait de Sicherbeit - qui demeure la motivation centrale de la théorie des démonstrations. Et si les mathématiques (et la logique) classiques assurent le confort théologique du paradis cantorien que Hilbert malgré tout ne voulait pas quitter, le constructivisme "athée» préfere encore les nourritures terrestres et le dur labeur de la vérification ou mieux de la «vérifaction» aux fictions idéales et au rêve figé d'un ciel incertain.

Département de philosophie Université de Montréal 